\title{
Applications for Children with Autism in Preschool and Primary Education
}

\author{
https://doi.org/10.3991/ijes.v7i2.10335
}

\author{
Maria Xanthopoulou $\left.{ }^{\varpi}\right)$, Georgia Kokkalia, Athanasios Drigas \\ National Center for Scientific Research-Demokritos, Attica, Greece \\ maxanthopoulou@yahoo.com
}

\begin{abstract}
In this article, we decided to focus on applications for children with Autism Spectrum Disorder (ASD) knowing the significant influence of them on those children. Below, we analyze the different types of applications that are available for children with ASD. The applications are presenting, have been categorized in three categories. The first category is Diagnostic Tools, the second is Intervention Tools and the third is generally Mobile Apps. This separation was made to give a clear view about the characteristics and the developmental area each application covers. Finally, the applications have been chosen, based on positive reviews, in combined with how many had download and install them, whether they were easy to use and if they responded to what their description said they were providing.
\end{abstract}

Keywords-ASD, Diagnostic Tools, Intervention Tools, Mobile Apps

\section{Introduction}

Autism characterized as a neurodevelopmental disorder that affects a child at a very early age, before three and last throughout the entire life. This disorder produces persistent deficiencies in social communication, difficulties in interaction and manifestation of repetitive or unusual behaviors. Autism belongs at ASD, which called "spectrum disorder" because of the symptoms that could be range from mild to severe. ASD also includes Asperger's Syndrome, Childhood Disintegrative disorder and Pervasive Developmental Disorder not otherwise specified (PDD-NOS) [2, 3, 18, 38].

According to current reports, the last few years is estimated that there is a $30 \%$ globally increase in children diagnosed with ASD. Countries with highest rate of autism are United States, China and South Korea. Autism also found in Asia, Europe and other regions $[2,47]$. About clinical diagnosis of ASD, is reported that typically occurs between the ages of 3 and 4 , and through surveys it seems that boys are more affected than girls $[2,38]$. The cause of ASD has not been clearly identified, but there are some significant causes, such as genetic problems, advanced age of the parents and the child's exposure to certain drugs during the pregnancy [41].

There are two main early signs of autism. The first is social un-connectedness such as reduced eye contact that can be observed already at the age of six months and the 
second is repetitive behaviours such as flapping hands [46, 48, 49]. Also there are more early indications of autism like pre aggressive behaviour such as, rocking head backward and forth, open mouth with tighten teeth and shrank eyes [49].

Despite extensive and comprehensive ASD researches, there are currently no medical laboratory tests to diagnose autism and is not curable [14, 44]. That's why is very important to diagnose autism as early as possible so there is early intervention to improve child's condition [14]. In many developing countries early diagnose and intervention services are unable to offer such intensive services to children with ASD, due to lack in trained staff and high costs of intensive therapy, because of their extended or indefinite duration. As a result, many families of children with autistic disorder have limited access in diagnose and early intensive behavioral intervention (EIBI) [38].

The importance of early intervention, lies in the fact that in early ages children have neuroplasticity, and through early diagnose and intervention they can develop and improve the areas in which they have difficulty [38]. In addition, current neurophysiological and behavior experiments, show that children with autism have lack of emotional empathy and because of that, it is difficult to them to share their feelings and recognize what the others feel. That's why children in the autistic spectrum, often have reduced or unexpected behavior, when they have to deal with their or other's emotions and that causes problems in their social relationships [57].

The appropriate efficient and effective intervention and training environment for children with autism, is provided by computers, tablets, smart phones etc., so they fulfill their needs as they have specific structure, consistency and predictability. That seems to help increase communication skills among children with ASD, by a way of augmentative alternative communication (AAC) or, on-screen social information [16, $22,38,57]$. This is one of the reasons that autistic children, are attracted by technology. Another reason which technology has high appeal to children with ASD, is the fact that most of these children are visual learners [38].

Furthermore, technology provides to those children a non-stress frame that is allowed them to learn with their own pace [14, 22, 57]. Also, using technology for early diagnose, intervention, and skills improving, we give the chance to families with autistic children in developing countries, where there is high cost of intensive therapy and lack of awareness, resources, services and trained professionals to administer autism, to have access to all the necessary procedures of diagnose, intervention and rehabilitation that will help their children [2].

\section{Diagnostic Tools for Children with Autism in Preschool and Primary Education}

Autism needs careful observation, so to be diagnose, different screening tools need to be used depending on the age of the children which may not be well adopted in all countries. Screening tools have been developed for children of different age group, social behavior, culture, customs, traditions and local context, as all of these varies from country to country. Many screening applications have been developed to screen 
autism automatically. However, till date there are no applications able to ensure interactive as well as efficient screening with clinical validation [2].

The applications that follow use interactive screening questionnaires, they have small age scope and the last one uses video assessment.

EarlyThree is a childhood development tracking tool, that is about children from 8 to 36 months. This app is very helpful for parents and pediatricians, as provide them the ability to detect developmental delays such as ASD. The users have to answer a series of questions about their child's development. At the end of the process they can review and edit their answers. Finally, the question's results are displayed in three different coloured bands. Green for normal development, Yellow for more watchful monitoring and Red for developmental delays and further medical evaluation [17].

ASDetect is evaluated the social and behavioral characteristics using videos and non-videos combined with questionnaires. Has designed for toddlers younger than 2.5 years and is a video-based app that parents can use to look for autistic traits related to their children. Therefore, children over 2.5 years of age, adolescents and adults cannot served by ASDetect [52].

Below we analyze another type of application in which the assessment process consists of interactive screening questionnaires but unlike previous applications, it covers a larger age scope.

sASDTest is an application offers a friendly, time-efficient and accessible autism tests and covers the entire age range from 18 months to adults. Also, it serves larger communities worldwide as well as being available in 11 different languages and maintained the brevity as each test contains 10 questions. The users selects the proper language and age category. Once the users complete the test and amend any of the answers they want, they automatically transferred to the results screen, where they will see the score number of their test with appropriate text. For toddlers, if the score is greater than 3 that denotes a clinical assessment being necessary. If the score is less than 3 there are no ASD traits. Also, the users can e-mail the results as PDF form that contains the necessary information [52].

The application is presented below has interactive screening questionnaires for ASD detection, covers a larger age scope and contains more assessment steps.

Smart Autism is an app that purposed to automate the traditional approaches to screening and confirming autism. This app has an integrated screening tool targeting different age group, from 0 to 17 years old, to speed up the process. Depending on the child's birth date the framework selects the appropriate screening method. It consists of three layers assessment process, Screening, Virtual Assessment and Actual Assessment. At Screening, if autism is suspected, the framework automatically refer the user to the Virtual Assessment process. The child watches a video and its reaction is recorded and uploaded to the cloud to remotely expert evaluation. At last, if autism is still suspected the integrated framework confirms autism automatically and referred the child to the nearest Autism Resource Center (ARC) for Actual Assessment [2, 50].

The most complete type of this category is the one mentioned below, which besides the features previous types of applications, has also Parent Counseling, and Intervention and Follow-up. 
AutismXpress it is an integrate cloud based framework that has the ability of screening, can track diagnostics results, also provides online counseling for the parents and can monitor intervention progress automatically. The age range it captured is from 0 to 17 years. Depending on the age the user enters, the app selects the appropriate set of questionnaires. The first app's section is Screening, which uses three different screening tools (M-Chat-R, Childhood Autism Spectrum Test and Autism Spectrum Screening Questionnaire). If responses show that the child has ASD then the follow section, Confirmation, is initiated, coordinated and tracked automatically by the cloud. It consists of Virtual and Actual Assessment. The child will see some videos with different activities in the Virtual Assessment process and depending on its response, it will be referred to the nearest ARC for Actual Assessment process and if autism is detected, an expert will suggest the appropriate plan for the child. As for the Parent Counseling section, videos, mostly about behavioral and educational aspect, are uploaded to a YouTube channel for parents. In the Intervention and Follow-up section, the child's progress is continuously tracked and monitored by the system, and that enables experts to make the necessary changes at child's diagnosis and skill development. However, Autism Express is underdevelopment but when is completed it would bring significant changes in the traditional autism diagnosis and treatment process [13].

The last category of diagnostic applications is that their assessment process has been based on analyze of recording children's reaction on stimulus to detect ASD traits. Below we present an app with the previous characteristics, that it also cover a large age scope.

Cognoa for child development is a diagnostic app that currently is only available for the parents as a family benefit through employers and in select physician's offices. The native app does not give the chance to other users to create account. Cognoa has digital developmental assessment, personalized therapeutics and expert activities. All these provide accurate, earlier diagnoses and more effective treatments. This app is about children from 1 to 8 years. Firstly, the parents has to answer a parental questionnaire which includes 17 or 21 questions, depending on the child's age. In addition, is requested by the parents to record a two or three minute home video of the child's behavior and uploaded so the experts have a more complete view of the child to do the diagnose. After this procedure the app informs the parents for the results $[1,6,21$, 29].

At the same category belongs Autism \& Beyond which is a video-based screening app, is available for the public but it is used for the study on child mental health of Duke University and the Duke Medical Center. The future goal is to be used by the public to diagnose ASD at home [11].

\section{Intervention Tools for Children with Autism in Preschool and Primary Education}

Data generated from surveys shows that EIBI is a very effective treatment for children with ASD [38]. Most researches for applications have focused on the study of 
them as intervention systems and not as intervention devices themselves. Due to the increase of children diagnosed with ASD at younger ages, coupled with the emphasis on early intervention, the demand for healing methods is increasing [47].

There are different categories of intervention applications for ASD. The two apps which are presented below, are apps that helps children to develop their developmental delays, improve their skills and mental disabilities.

TOBY (Therapy Outcomes by You) is an intervention app that has the key features of a comprehensive, individualized and quality EIBI. TOBY is a platform based-on applications that provides timely intensive behavioral interventions to facilitate learning for children with ASD $[38,55]$. TOBY actually teach parents the basics of operant conditioning so they can help their children at the crucial areas of their development. This app can be used by children or parents at any time and conjunction with other early intervention programs. It has three types of tasks. The first type is the Solo task which can performed by the child without assistance but it doesn't cover a wide range of skills. The algorithm will prompt the child if wrong and reward it if correct. The second type is Partner task which provide parents oversee their child's progress. At the third type of tasks, Natural Environment Training, skills are transferred to natural settings to become generalized. The app contains four skills areas: Sensory, Imitation, Language and Social. At last, the app provides parents an activity report, where they can monitor their child's progress, by seeing the skills that have been mastered which are grouped by skills areas [56].

Autism early intervention - Cuedin app is an android based early intervention educational app for slow learners, children with special needs, developmental delays and mental disabilities. The age range it covers is from 3 to 9 years old [51]. It has Manage Sensory Overload by limited using of colors and sounds, which provides a very predictable sensory input and a comfortable learning experience. The app tries to provide a framework that includes a wide range of development areas such as academics, languages, social skills, everyday routines, etc. The user can print worksheets from the vast connect database. In addition, is able to track and record user's performance to see if the objective target is met. Finally, is about an app that is continuously improving to provide as many options as possible [25, 51].

Another category of intervention apps is that deals with social and emotional delays and help children with autism to improve their empathy, social behavior and relationships with others.

CaptureMyEmotion is an app still in using only for research and it's not available for the public yet. However, is important to mention it because, this app uses sensor input to measure the user's emotions and helps users gauge the intensity of their emotions. The app's target group is children from 7 years and older who are capable of using a smartphone. Is about a personalized tool for children that provides them the chance to learn, understand and discuss their feelings. Children are able, at the same time, to take photos, videos, sounds, sense and self-report their emotions. CaptureMyEmotion uses a wrist worn sensor called "Q $\mathrm{Q}$ sensor" which measures skin temperature and motion changes. The sensor it's easy to worn, transmits the data in real time to a mobile phone, can be worn for a long period of time and gives an indication about the wearer's emotional state. Finally, based on the physiological data are col- 
lected the app calculates the arousal level, which is shown under each picture, video or sound together with other information such as date, time and GPS coordinates [19, 42].

My digital problem solver (DPS) is aimed to 9-12 years children. Is about an app that helps users to identify emotions and coping strategies. The app enables children to select a feeling and a coping strategy in order to manage themselves and calm down. My DPS features eight feelings which are Bored, Surprised, Mad, Annoyed, Happy, Scared, Sad and Frustrated. Also the app includes four coping strategies which are "Use Positive Self-Talk", "Take Five Deep Breaths", "Take a Break to Calm Down" and "Remind Myself to Keep Negative Thoughts in My Head". Every time the user selects a strategy will see a Hollywood quality animated video which show the user the way to do the strategy. Additionally, the users can add their own feelings and coping strategies by taking photos or importing pictures from their phone. Finally, users can add text to label the feeling or the strategy [35, 53].

In the same category there are some other apps for emotional and social improvement in children with ASD such as Sesame Street Breathe, Pocoyo Playset-Feelings, Expressions for Autism [20], Avokiddo Emotions [12], ABA Flash Cards \& Games - Emotions, Touch and Learn - Emotions [39, 54], Learn with Rufus: Emotions [53] etc.

Another important category is that of applications for communication support and speaking and learning ability improving. Some of them are presented below.

Otsimo is a certified and awarded educational game application which is based on ABA therapy, one of the most widely known and trusted behavioral therapy technique for children with learning disorders and attention deficit problems. The app is aimed to teach fundamental education about core skills such as numbers, emotions, words, etc. Otsimo has a free AAC for nonverbal children or with speech problem. The game has two separate platforms for children and their families. Children section includes ad-free educational games. Family section is a platform where the parents can have access to their child's education program, they can see review of their child's progress, checks the reports and configure difficult settings. Finally, Otsimo provides access to more than 50 educational games, personalized special education for each child, daily and weekly reports about the games the child played [36].

Autism Language and Cognitive Therapy with MITA is an early intervention computerized brain training application for children with ASD. Children from 5 years and under can use the application. The app is based on ABA technique of visual-visual and auditory-visual conditional discrimination, Language therapy technique of following directions with increasing complexity and Pivotal Response Treatment that targets on response development to multiple cues. MITA has available 9 different types of games: Outlines (easy), Patches (easy/intermediate/advanced), Matching (easy), Wooden Puzzles (easy), Odd-one-out (intermediate), Elephant Combo (intermediate), Toy Combo (advanced), Analogies (advanced) and Language Game (intermediate \& advanced). All the games aim to help children learn how to mentally integrate, notice and report multiple visual cues presented simultaneously at the features of an object. In addition, each activity is adapted at the child's development level, there are animated characters and Playtime rewards that keep child's attention, the 
interface is clear and intuitive, also there are no ads and Wi-Fi is not necessary to use the app $[26,40]$.

In the same category there are some other apps such as, I Can Communicate, Symbo Talk, My Talker AAC, Leeloo AAC, AAC Autism myVoiceCommunicator [24], Proloquo2Go - Symbol-based AAC, Avaz Pro - AAC App for Autism, TouchChat HD - Lite [39], Card Talk [27], Let me talk [8][34][24] Endless reader [7, 30], ABC Kids - Tracing \& Phonics [4, 24], Autism Read \& Write, Autism Read \& Write Pro [24] etc.

The app is follow is a comprehensive tool supporting the therapy for children with developmental and behavior disorders, such as autism, Down Syndrome, ADHD, mental disability, celebral palsy and others.

Happy Therapy is a comprehensive tool for developmental or behavior disorders therapy. The application has two variants. Happy therapy Home and Happy therapy $P R O$. The first variant, Happy therapy Home, contains games that support the therapy, the child's progress monitoring system with the statistics and also gives the users the ability to choose the game they think that is suitable for the child. The second variant, Happy therapy PRO, gives to the therapist the option to create profiles for several children. Also contains the same games and monitoring system with the first variant with the difference that monitoring system has more extending statistics, contains organizer and gives the ability of communication between therapists and the option of customizing the games to each child [31].

\section{Mobile Apps for Children with Autism in Preschool and Primary Education}

Below we are presenting some other applications are not related to diagnose or intervention, but they are also very useful to families with children in autistic spectrum. They help children to deal with daily situations and improve their abilities. The first app provide parents guidance for their children.

Naturalistic Observation Diagnostic Assessment (NODA) is a medical researchbased mobile app. Is used for children from 18 months to 7 years. The application's purpose was to provide parents the proper guidance on autism traits. Users, which could be, parents, caregivers, teachers or clinicians, record and upload a video of their children and the experts can provide comments. Special technology is using to collect behavior information as "behavior specimens" from child's natural environment. Finally, the app provide a report at the user that medical experts can use during the diagnosis process $[9,15,52]$.

The apps are presented below help children with ASD to organize their daily schedule and cores, and improve socially appropriate behavior.

Children with autism is a visual schedule app. It is the first wearable picture-based scheduler. Children 4 years and older can use the application. The app's user-friendly interface provides an easy way to the users to create their own picture-based, personalized daily schedule. Children with autism displays how much time has passed and when the event will end through icons and progress bar, helps the users concentrate in 
order to complete their tasks, checks the status of a current event and is able to review all the tasks are related with the event. Finally, the users can create and edit daily and weekly schedules with simple drag and drop, either with the 14 different event icons the app contains for the school and home, and either with the icons they can create using photos $[5,45]$.

Kids ToDo List is an app for visual schedule. The app is developed based on LITALICO classrooms and aimed at children up to 8 years. There are 100 types of cards that are supported by voice sounds in multiple languages. However, the users can create original picture cards adding photos from their phone or taking new with their camera and recording their voice. Users can easily create their own visual schedule with the tasks they had to do during the day by scribbling the cards. Finally, when a user complete a task he takes a reward, such as tapping stars, collecting fish etc. [33].

Kids task timer - visual timer for kids is a timer for kids up to 8 years. The app helps them to complete their daily routine tasks and manage the time for each tasks, as at those ages children are confusing with the meaning of the time and they cannot read yet the clock. The app provides audio and visual alerts that helps them to follow their schedule properly [32].

ChoreMonster is an application that makes children's daily chores such as brushing their teeth, eating, cleaning their bedroom etc. a fun process. The app can be used by children from 4 to 12 years. When they finish their chores, they earn points and rewards to use them for things like playing games on the tablet, getting to the movies or eating candies. Also, the chores that children are called to do and the rewards can earn are managed by their parents [28].

Visual Schedules and Social Stories is a visual support app that uses social stories and visual schedule to help children with autism to improve their social behaviour. This is an app can be used by teachers, parents, individuals with autism and communication or neurotypical challenges and special educators. Children from 6 to 12 years can use the application. To make their own visual schedule and social stories, users can use the program's cartoon pictures and text. Also, they are able to upload their own images, video and audios. Users have the option to share, email and print their schedules and social stories.

Finally, it is easy to use, it has attractive interface, there are no ads and does not require internet [37].

Social adventures is aimed to children from 3-13 years. Children with ASD using the app can improve their social skills and relationships. Through this app children taught how to initiate and maintain interactions, how to advocate, how to get regulated, how to interpret non-verbals, how to negotiate their personal space and experience humor. Social adventures includes 80 activities that can be used in groups, classrooms, play dates and everywhere else. There are step by step instructions and a section with tips for parents, generalized social phrases, visuals to be used for cue cards or role paly and a sample of 8 -week program. Also is a self-contained resource, as each activity can be used to augment other approaches [10, 54].

In the same category there are many other application such as, TimeTune - Optimize Your Time, Happy Kids Timer-Morning \& Evening Chores, Timo: Kids Routine 
to Practice Time Management, On Timy - planer for children, Pictogram Agenda, First Then, Visual Schedules and Social Stories, App4Autism, Autism Aid App [24] etc.

Find My Family, Friends \& iPhone - Life360 Locator is an app that helps children with autism and their parents, to follow their schedule, see where their parents are at real time, text any time to them and emit SOS signal if they are in danger. Also, is a very helpful app for parents with autistic children because they can check, at real time, where their child are, when they leave school, where they go next, they can text each other and be in touched all day [43].

There are several other apps that help and support children with ASD and their families. Indicatively we present some more which belong at the top lists of the apps for autism and which have high rates at Google play and App Store. Some of them are: Autism Parenting Magazine, Sesame Street and Autism, Sensory Baby Toddler Learning, Kinder tangram: Build a House [39]. There are also some applications, such as Calm, Stop, Breath \& Think, Mitra, Smiling Mind and Mood Meter, that are very useful because they help autistic children calm down, manage the situations that make them nervous and cause them anxiety, control and understand their feelings [54]. Finally, there are apps that give parents the ability to keep an eye on their children all day and know exactly where they are such as, Family Locator, Family Locator by Fameelee, Find My Friends \& Family by iSharing, Family GPS Locator for your Phone - Safe365, GeoLocator Parental Control, Child Safety Location, Family Locator - GPS Tracker [24] etc.

\section{Conclusion}

The development of technology into the field of ASD treatment and touch screen tablets has reimagined the portability, affordability, accessibility, social acceptance of obtaining information and education via technology [38]. However, there is no formal guidance in selecting and using applications. Study results show that even the most popular applications have defects that limits their educational usefulness. For that reason users are advised to carefully monitor applications before using them with children [47]. Finally, in its most optimal form, an application that intends to teach basic relational concepts to children with ASD should follow best practice strategies from early intensive behavioral intervention.

This article's importance is that presents a wide range of applications for children in preschool and primary education, about early diagnose and intervention and other kind of apps equally helpful and that all the applications have been categorized by their characteristics. In future researches we hope to see an even more specific and detailed categorize, analysis and description of applications for children with ASD.

\section{$6 \quad$ References}

[1] Abbas, H., Glover, E., \& Wall, P. D. (2017). Machine learning for early detection of autism (and other conditions) using a parental questionnaire and home video screening. $\mathrm{Pa}$ - 
per presented at IEEE International Conference on Big Data (BIGDATA) (pp. 3558-3561). Boston, MA: USA. https://doi.org/10.1109/bigdata.2017.8258346

[2] Al Mamun, K. A., Bardhan, S., Ullah, Md. A., Anagnostou, E., Brian, J., Akhter, S. \& Rabbani, G. M. (2016). Smart Autism - A mobile, interactive and integrated framework for screening and confirmation of autism. Proceedings of the 38th Annual International Conference. Paper presented at IEEE Engineering in Medicine and Biology Society (EMBC) (pp. 5989-5992). Orlando, Florida, USA. https://doi.org/10.1109/embc.2016.7592093

[3] American Psychiatric Association (2017). DSM-5 Fact Sheets. Accessed at 13 Jan 2019. Available in: https:/www.psychiatry.org/psychiatrists/practice/dsm/educationalresources/dsm-5-fact-sheets

[4] App Store Preview (2013a). ABC Kids - Tracing \& Phonics. Accessed at 20 Jan 2019. Available in: https://itunes.apple.com/us/app/abc-kids-tracingphonics/id1112482869? $\mathrm{mt}=8$

[5] App Store Preview (2015). Children with autism: A visual schedule. Accessed at 5 Dec 2018. Available in: https:/itunes.apple.com/us/app/children-autism-visualschedule/id1037037909? $\mathrm{mt}=8$

[6] App Store Preview (2019). Cognoa for Child Development. Accessed at 10 Feb 2019. Available in: $\quad$ https://itunes.apple.com/us/app/cognoa-for-childdevelopment/id965275481? $\mathrm{mt}=8$

[7] App Store Preview (2013b). Endless reader. Accessed at 20 Jan 2019. Available in: https://itunes.apple.com/gr/app/endless-reader/id722910739? $\mathrm{mt}=8$

[8] App Store Preview (2014a). Let me talk. Accessed at 20 Jan 2019. Available in: https://itunes.apple.com/us/app/letmetalk/id919990138?mt=8

[9] App Store Preview (2014b). NODA Autism Diagnosis. Accessed at 7 Feb 2019. Available in: https://itunes.apple.com/us/app/noda-autism-diagnosis/id1179502661? mt=8

[10] App Store Preview (2011). Social adventures. Accessed at 18 Jan 2019. Available in: https://itunes.apple.com/us/app/social-adventures/id468235375? $\mathrm{mt}=8$

[11] Autism and Beyond (2015). Autism and Beyond. Accessed at 21 Nov 2018. Available in: https://autismandbeyond.researchkit.duke.edu/ch

[12] Avokiddo (2019). Avokiddo emotions. Accessed at 7 Feb 2019. Available in: https://avokiddo.com/apps/avokiddo-emotions-app/ https://doi.org/10.20361/dr29383

[13] Bardhan, S., Md. Ullah, A., Ahmed, H. U., Rabbani, M. G., \& Al Mamun, K. A. (2016). Autism Express. A cloud based framework for autism screening, confirmation and intervention. Proceedings of the International Conference. Paper presented at IEEE Region 10 $\begin{array}{llll}\text { Conference (TENCON) (pp. } & \text { 414-419). }\end{array}$ https://doi.org/10.1109/tencon.2016.7848032

[14] Barton, M. L., Dumont-Mathieu, T., \& Fein, D. (2012). Screening Young Children for Autism Spectrum Disorders in Primary Practice. Journal of Autism and Developmental Disorders, 42(6), 1165-1174. https://doi.org/10.1007/s10803-011-1343-5

[15] Behavioral imaging (2018). How NODA works. Accessed at 13 Jan 2019. Available in: https://behaviorimaging.com/noda-howitworks/

[16] Bölte, S., Golan, O., Goodwin, M. S., \& Zwaigenbaum, L. (2010). What can innovative technologies do for Autism Spectrum Disorders? Autism, 14(3), 155-159. https://doi.org/10.1177/1362361310365028

[17] EarlyThree (2012). EarlyThree. Accessed at 26 Nov 2018. Available in: http://earlythree.com/

[18] El Kaliouby, R., Picard, R., \& Cohen, S. B. (2006). Affective Computing and Autism. Annals New York Academy of Sciences, 1093, 228-248. https://doi.org/10.1196/annals.1382.016 
[19] Gay, V., Leijdekkers, P., Agcanas, J., Wong, F., \& Wu, Q. (2013). CaptureMyEmotion: Helping Autistic Children Understand Their Emotions Using Facial Expression Recognition and Mobile Technologies. Proceedings of the 26th Bled Conference - "Challenges and Impacts for Individuals, Organizations and Society»-(pp. 1-12). Bled, Slovenia.

[20] GeekSPL (2017). Favorite apps for teaching feelings and emotions. Accessed at 18 Jan 2019. Available in: http://www.geekslp.com/2017/05/favorite-apps-for-teachingfeelings-and-emotions/

[21] Globe Newswire (2018). Cognoa. Accessed at 1 Dec 2018. Available in: http://www.globenewswire.com/news-release/2018/06/12/1520417/0/en/SAP-Teams-withCognoa-to-Bridge-Gap-in-Healthcare-for-Employees-Raising-Young-Children.html

[22] Golan, O., \& Baron-Cohen, S. (2006). Systemizing empathy: Teaching adults with Asperger syndrome or high-functioning autism to recognize complex emotions using interactive multimedia. Development and Psychopathology, 18(2), 591-617. https://doi.org/10.1017/s0954579406060305

[23] Google play (2018a). ABC Kids - Tracing \& Phonics. Accessed at 18 Jan 2019. Available in:

https://play.google.com/store/apps/details?id=com.rvappstudios.abc kids toddler tracing phonics\&hl=en

[24] Google Play (2018b). Apps. Accessed at 4 Dec 2018. Available in: https://play.google.com/store/search?q=apps+for+autism\&c=apps. https://doi.org/10.7717/peerj.4849/table-6

[25] Google Play (2018c). Autism early intervention app. Accessed at 3 December 2018. Available in: https://play.google.com/store/apps/details?id=com.learnup.cuedin\&hl=en

[26] Google Play (2018d). Autism language and cognitive therapy with MITA. Accessed at 5 Dec 2018. Available in: https://play.google.com/store/apps/details?id=com.imagiration.mita

[27] Google Play (2018e). Card Talk. Accessed at 4 Dec 2018. Available in: https://play.google.com/store/apps/details?id=ip.co.litalico.cardtalk

[28] Google Play (2018f). ChoreMonster. Accessed at 7 Dec 2018. Available in: https://play.google.com/store/apps/details?id=com.choremonster.cmandroid

[29] Google Play (2019). Cognoa for Child Development. Accessed at 15 Jan 2019. Available in: https://play.google.com/store/apps/details?id=com.cognoa.androidclient\&hl=en

[30] Google Play (2018g). Endless reader. Accessed at 17 Jan 2019 Available in: https://play.google.com/store/apps/details?id=com.originatorkids.EndlessReader\&hl=en

[31] Google play (2016). Happy therapy. Accessed at 17 Jan 2019 Available in: https:/play.google.com/store/apps/details?id=com.dromnibus.dromnibus\&hl=en

[32] Google Play (2018h). Kids task timer - visual timer for kids. Accessed at 7 Dec 2018. Available in: https://play.google.com/store/apps/details?id=com.digit.kidstimer

[33] Google Play (2017a). Kids ToDo List. Accessed at 6 Dec 2018. Available in: https://play.google.com/store/apps/details?id=jp.co.litalico.kidstodolist

[34] Google Play (2017b). LetMeTalk: Free AAC Talker. Accessed at 4 Dec 2018. Available in: https://play.google.com/store/apps/details?id=de.appnotize.letmetalk

[35] Google Play (2012). My digital problem solving. Accessed at 17 Jan 2019 Available in: https://play.google.com/store/apps/details?id=com.thelanguageexpress.thesocialexpress.m ydps\&hl=en

[36] Google Play (2018i). Otsimo - special education ABA therapy autism game. Accessed at 5 Dec 2018. Available in: https://play.google.com/store/apps/details?id=com.otsimo.app 
[37] Google Play (2018j). Visual Schedules and Social Stories. Accessed at 7 Dec 2018. Available

https://play.google.com/store/apps/details?id=com.mts.visualscheduleandstorymaker

[38] Granich, J., Dass, A., Busacca, M., Moore, D., Anderson, A., Venkatesh, S., Duong, T., Vellanki, P., Richdale, A., Trembath, D., Cairns, D., Marshall, W., Rodwell, T., Rayner1, M., \& Whitehouse, A. J. O. (2016). Randomised controlled trial of an iPad based early intervention for autism: TOBY playpad study protocol. BMC Pediatrics, 16(1), 1-12. https://doi.org/10.1186/s12887-016-0704-9

[39] Health line (2017). The Best Autism Apps of the Year. Accessed at 7 Dec 2018. Available in: https://www.healthline.com/health/autism/top-iphone-android-apps

[40] ImagiRation (2018). General questions. Accessed at 5 Dec 2018. Available in: http://imagiration.com/autism/mita-faqs/

[41] Landrigan, P. J. (2010). What causes autism? Exploring the environmental contribution. $\begin{array}{llll}\text { Current Option in } & \text { 22, } & \\ \end{array}$ https://doi.org/10.1097/mop.0b013e328336eb9a

[42] Leijdekkers, P., Gay, V., \& Wong, F. (2013). CaptureMyEmotion: A mobile app to improve emotion learning for autistic children using sensors. Proceedings of the 26th IEEE International Symposium on Computer-Based Medical Systems (pp. 381-384). Porto, Portugal. https://doi.org/10.1109/cbms.2013.6627821

[43] Life360 (2019). Accessed at 5 Dec 2018. Available in: https://www.life360.com/

[44] Lord, C., Risi, S., DiLavore, P. S., Shulman, C., Thurm, A., \& Pickles, A.(2006). Autism from 2 to 9 years of age. Arch Gen Psychiatry, 63, 694-701. https://doi.org/10.1001/archpsyc.63.6.694

[45] Medcalf, L. (2018). Children with autism: A visual schedule app. Accessed at 4 Dec 2018. Available in: https://www.eastersealstech.com/2018/01/30/children-autism-visualschedule-app/

[46] Miller-Wilson, K. (2017). Hand Flapping. Love to know. Accessed at 16 Nov 2018. Available in: https://autism.lovetoknow.com/diagnosing-autism/hand-flapping

[47] Mykyta, A. D. \& Zhou, Z. (2017). Accessing quality apps to promote basic relational concepts acquisition among young children with autism. Wiley, 54, 1302-1311. https://doi.org/10.1002/pits.22082

[48] Perera, H., Jeewandara, C., Guruge, C., \& Seneviratne, S. (2013). Presenting symptoms of autism in Sri Lanka: Analysis of a clinical cohort. Sri Lanka Journal of Child Health, 42(3). https://doi.org/10.4038/slich.v42i3.6017

[49] Q and A: Head Banging Solution (2014, Mai 30). Autism Parenting Magazine, 18.

[50] Smart ASD (2019). Smart-ASD App. Accessed at 7 February 2019. Available in: https://smart-asd.eu/researchers-and-teachers/smart-asd-app

[51] Social App Hub (2017). Autism early intervention app. Accessed at 3 Dec 2018. Available in: https://www.socialapphub.com/app/autism-early-intervention-app

[52] Thabtah, F. (2018). An accessible and efficient autism screening method for behavioural data and predictive analyses. Health Informatics Journal, 1-17. https://doi.org/10.1177/1460458218796636

[53] The social express (2016). Amazing apps for autism you absolutely need to try. Accessed at 17 Jan 2019 Available in: http://thesocialexpress.com/amazing-apps-autism-absolutelyneed-try $/$

[54] The tech edvocate (2017). 8 Must-have emotional intelligence apps and tools. Accessed at 18 Jan 2019. Available in: https://www.thetechedvocate.org/8-must-emotionalintelligence-apps-tools/ 
Paper-Applications for Children with Autism in Preschool and Primary Education

[55] TOBY Autism Therapy (2016). TOBY autism therapy (therapy outcomes by you). Accessed at 13 Jan 2019. Available in: http://tobyplaypad.com/

[56] Venkatesh, S., Phung, D., Duong, T., Greenhill, S., \& Adams, B. (2013). TOBY: Early Intervention in Autism through Technology. Proceedings of the SIGCHI Conference on Human Factors in Computing System (CHI). Changing Perspectives (pp. 3187-3196). Paris: France. https://doi.org/10.1145/2470654.2466437

[57] Zoerner, D., Schütze, J., Lucke, U., Kirst, S., \& Dziobek, I. (2016). Zirkus Empathiko. Mobile Training of Socio-Emotional Competences for Children with Autism. Paper presented at IEEE $16^{\text {th }}$ International Conference on Advanced Learning Technologies (pp. 448-452). Austin, TX: USA. https://doi.org/10.1109/icalt.2016.146

\section{Authors}

Maria Xanthopoulou graduated with excellent from the Department of Early Childhood Education at the National and Kapodistrian University of Athens (NKUA). She is a Special Education Teacher and postgraduate student in the field of Comparative Education, Educational Politics and Administration of Education at NKUA. She is associated with N.C.S.R. 'Demokritos', Institute of Informatics and Telecommunications, Net Media Lab \& Brain-Mind R\&D, Agia Paraskevi, 153 10, Athens, Greece (e-mail: maxanthopoulou@yahoo.com).

Georgia Kokkalia (MSc in Specific Learning Difficulties) is a Special Education Teaching Professional and Phd Candidate in University of Athens. She has participated in various research projects regarding the use of Information and Communication Technologies (ICTs) in Special Education and in Kindergarten.Also is with N.C.S.R. 'Demokritos', Institute of Informatics and Telecommunications, Net Media Lab \& Brain-Mind R\&D, (e- mail: gioulina@hotmail.com).

Athanasios Drigas is a Director of Research in N.C.S.R. 'Demokritos', Institute of Informatics and Telecommunications, and Coordinator of Net Media Lab \& BrainMind R\&D, Agia Paraskevi, 153 10, Athens, Greece (e-mail: dr@iit.demokritos.gr).

Article submitted 2019-02-18. Resubmitted 2019-04-18. Final acceptance 2019-04-28. Final version published as submitted by the authors 